\title{
Aproximación a la orientación al mercado y sus consecuencias en unidades básicas de actuación pública: el caso del servicio de fomento de la economía social vasca
}

\author{
Jon Morandeira Arca \\ Profesor Adjunto \\ Departamento de Economía Financiera II (UPV/EHU) \\ Victoria de Elizagarate Gutiérrez \\ Profesora Colaboradora Honorífica \\ Instituto de Economía Aplicada a la Empresa - IEAE (UPV/EHU) \\ Baleren BAKaikoa AzURMEndi \\ Profesor Colaborador Honorífico \\ Departamento de Economía Aplicada I (UPV/EHU)
}

DOI: $10.1387 /$ reves. 17532

Fecha de entrada: 03/12/2015

Fecha de aceptación: 29/03/2016

Sumario: 1. Introducción. 2. Orientación al mercado en el Sector Público: Orientación al Servicio del Público. 3. Estudio de caso. 4. Discusión e implicación. Bibliografía.

\section{Resumen:}

La mayor parte de los estudios realizados, sino todos, en materia de orientación al servicio del público se han centrado en el ámbito institucional o nivel jerárquico de decisión estratégica. Por ello y teniendo en cuenta que la orientación al mercado puede variar entre distintos niveles de una misma organización, este trabajo tiene como objetivo el análisis de la orientación al servicio del público en una unidad básica de actuación pública dentro de un Gobierno, es decir, en un nivel jerárquico de la esfera táctica y operativa de la actuación pública. El análisis de la orientación al servicio del público y sus consecuencias en el rendimiento, se ha realizado sobre la unidad básica de actuación pública de fomento de la Economía Social del País Vasco. 


\title{
Palabras clave:
}

Orientación al mercado, marketing público, gestión pública, unidades básicas de actuación pública.

\begin{abstract}
:
The most, if not all, of the studies carried out in the field of public service orientation were focused on an institutional level or a hierarchical level of strategic decision and bearing in mind that market orientation can vary between different levels of the same organization, this work has concentrated on the analysis of public service orientation in a basic unit of public action within a Government, that is, on a hierarchical level of the tactical and operational sphere of public action. The analysis of public service orientation and its consequences was carried out on the basic unit of public action for development of the Social Economy of the Basque Country.
\end{abstract}

\section{Keywords:}

Market orientation, public marketing, public management, basic units of public action.

\section{Claves Econlit:}

H410, H830, M310

\section{Introducción}

El marketing como filosofía es una postura mental, una actitud, una forma de concebir la relación de intercambio entre dos o más partes (Santesmases, 2012), es decir, una filosofía que orienta a la organización al conocimiento y comprensión de sus clientes para la satisfacción de sus necesidades. Una filosofía donde la adopción del concepto de marketing implica la consideración de las necesidades del público en los procesos de elaboración de los proyectos públicos de forma que a través de la participación se transforma y adapta tanto la organización como los servicios ofertados (orientación al marketing/mercado) (Cervera, 2001). Por lo tanto, el marketing resulta ser la mejor plataforma de planificación para un organismo público que quiere satisfacer las necesidades de los ciudadanos y proveer auténtico valor. La principal preocupación del marketing consiste en ofrecer resultados valorados por el mercado objeto. En el sector público, el mantra del marketing es el valor para el ciudadano y su satisfacción (Kotler y Lee, 2007: 18). 
«En su capacidad de afrontar nuevos objetos de estudio ${ }^{1}$, el carácter interdisciplinar del marketing permite importar las teorías, conceptos y leyes de disciplinas conexas y estudiar objetos ya sujetos a investigación bajo una óptica distinta» (Cervera, 1999). Aunque la expansión del marketing en el sector público sigue siendo cuestionada o considerada periférica (Walsh, 1994), esta expansión es un hecho generalmente reconocido siendo numerosos los trabajos presentados sobre diversas aplicaciones del marketing en este campo.

En España, la aplicación del marketing en el sector público es mayoritariamente aceptada por los estudiosos del marketing (Ortega, 1977; Martín, 1993; Miquel et al, 1995; Chías, 1995 y 2007; Santesmases, 2012). Aunque son muchos los autores que desde disciplinas como el Derecho Administrativo abogan por el marketing (Ramió, 1999), por parte de otros la aplicación del marketing se percibe con ciertas reservas (Röber, 1989).

En este contexto, el núcleo central del marketing público es la gestión de la relación de intercambio que se mantiene entre la administración y el administrado, donde los componentes principales son los actores y los flujos entre los mismos. Los actores son, por un lado, la administración, y por otro, el administrado/ciudadano/usuario/cliente ${ }^{2}$. Considerando a la Administración Pública desde la Teoría General de Sistemas (Boulding, 1956), es decir, como un conjunto de subsistemas interrelacionados que coexisten dentro de un único sistema organizacional que, a su vez, se relaciona con otros sistemas del entorno, se observa que el análisis consiste esencialmente en distinguir entidades y definir los flujos que las une (Laufer y Burlaud, 1989). Dichos flujos son consecuencia de los diferentes intercambios que surgen entre la Administración Pública y el público.

Por ello, resulta de vital importancia la definición de los intercambios que se producen en el seno de la relación entre ambos. Chias (1995), de-

1 Esta línea de pensamiento se plantea la existencia de un marketing no empresarial como área específica dentro de la disciplina, que a su vez está conformado por subáreas de marketing no lucrativo, marketing público, marketing social, y marketing político y electoral (véase, por ejemplo, Santesmases, 2012 o Rufín y Medina, 2012).

2 El concepto de cliente de los ciudadanos debe ser entendido como expresión de mayor exigencia, activismo por ambas partes, e incorporando no solamente personas o individuos sino grupos, asociaciones o colectivos (Subirats, 1992). El cambio en el modelo de gestión del sector público europeo, tanto a nivel del Estado como a nivel de las corporaciones locales, se ha traducido en la promoción de los servicios públicos, considerando a los usuarios no como administradores, sino como clientes (Bouinot y Bermils, 1995). En España se ha tendido hacia el vocablo cliente, especialmente en los procesos de modernización de la Administración Pública (Chias, 1995). 
fine la gestión del marketing en el sector público como el proceso de planificación y ejecución de la política del Gobierno para crear intercambios (intercambios públicos) que satisfagan tanto los objetivos sociales y como los del Gobierno. Plantea un modelo general de intercambios, los llamados intercambios públicos, en el que se diferencian seis tipos de intercambios que se muestra en la Figura 1.1.

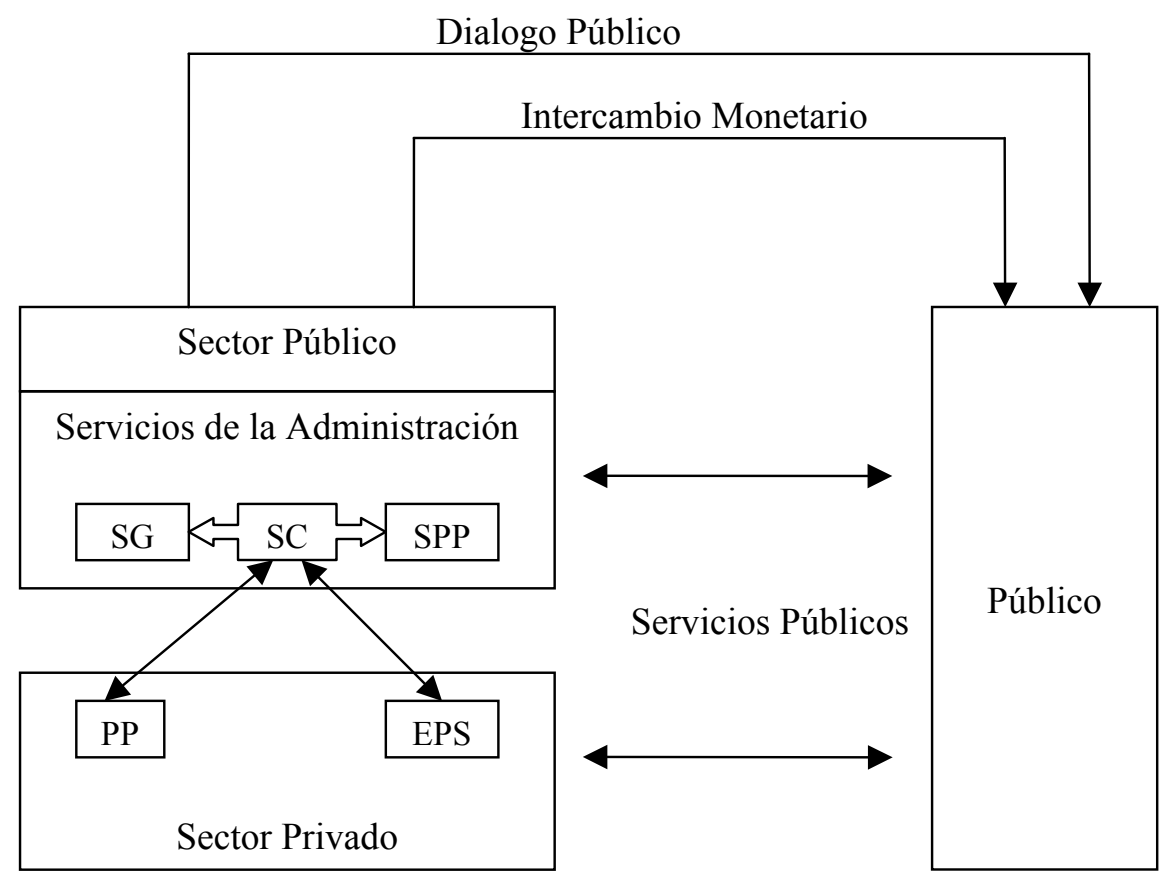

Fuente: Adaptado Chias (1995: 59).

Figura 1.1

Intercambios Públicos

Destacando, el intercambio de Servicios (véase Figura 1.2), en el que el Gobierno actúa como prestador de los servicios y el público a los que se dirigen soportan unos coste de participación que pueden ser:, por un lado, monetarios en el caso que se realice un pago directo de impuestos, tasas,.... (precio); y por otro lado, no monetarios en los que se encuentra el coste de 
accesibilidad (espacial, temporal y mental) y de participación en el proceso de prestación/producción.

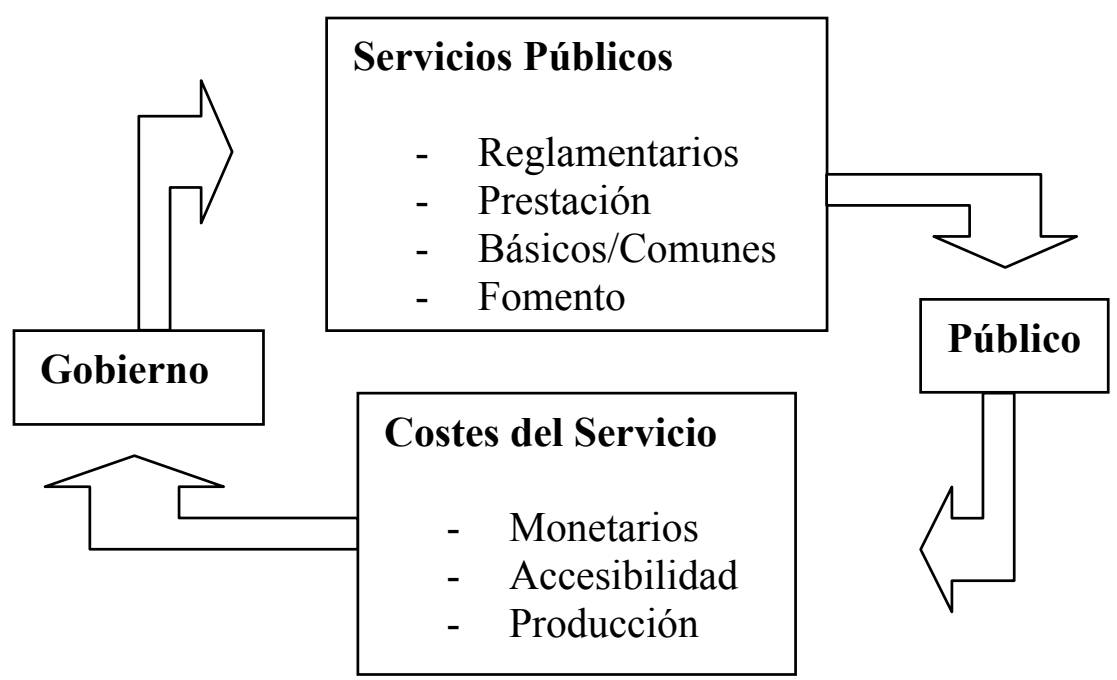

Fuente: Chias (1995: 52).

Figura 1.2

El Intercambio de Servicio Públicos

Aceptar la aplicabilidad del concepto de marketing en los gobiernos debe hacerse sin menosprecio de los diversos factores diferenciadores que modifican el funcionamiento del marketing en este entorno, de la oferta, de la relación gobierno-ciudadanos, de los productos ofertados por la administración y respeto al propio marketing (Ritchie y La Bréque, 1975; Laufer, 1976; Lamb, 1987; Snavely, 1991; Cowell, 1991). De hecho, al considerarse como un sistemas en constante interacción con su entorno económico y social, las organizaciones públicas no pueden limitar su relación con el público a la mera prestación sino que deben relacionarse con toda la sociedad frente a la que debe legitimar su existencia (Laufer y Burlaud, 1989).

Por ello, se debiera diferenciar entre el marketing de institucional, de relación o "de la caja negra» y el marketing de producto (Laufer y Burlaud, 1989). En este mismo sentido Vázquez y Placer (2000), seña- 
lan que el marketing institucional forma parte del marketing público como aquel que debe legitimar la actividad de la Administración Pública en su conjunto, junto con el marketing de servicios públicos que se centra en la relación de intercambio entre el público y un servicio público concreto. En este mismo sentido, Chias (1995 y 2007) también considera esa misma distinción cuando plantea un marketing público formado por el marketing-mix de gobierno y el marketing-mix de gestión.

Así, podríamos afirmar que el Marketing Público o de la Administración Pública está compuesta por dos aplicaciones de marketing:

- Marketing Público Institucional, aquél que se centra en el intercambio de dialogo público. De esta manera se provee la capacidad de agregar los distintos programas mediante una planificación de la actividad administrativa basada en criterios de transparencia y racionalidad (Röber, 1989), y por tanto, facilitando su legitimación en el entorno en que se ubica (Martín, 1993: 661).

- Marketing de Servicios Públicos, aquél que se centra en el intercambio de servicios públicos, facilitando la legitimación de la Administración Pública mediante los productos/servicios que oferta.

Niveles de marketing que no se dan de forma aislada y/o antagónica, sino que coexisten, si bien en distintos niveles, entre ellos en función de la capacidad de concretar las acciones en productos tangibles (Laufer y Burlaud, 1989; y Váquez y Placer, 2000).

\section{Orientación al mercado en el Sector Público: Orientación al Servicio del Público}

La asunción o la implantación de la filosofía de marketing implica la existencia o necesidad de asumir una orientación al mercado, de hecho, se define la orientación al mercado como la adopción del concepto de marketing (Grönroos, 1989 y 1994; Kohli y Jaworski, 1990) o como la operacionalización (McCarthy y Perreault, 1993).

Una de las primeras aplicaciones empíricas de la orientación al mercado en la empresa lucrativa fue la realizada por Narver y Slater (1990), quienes incluyen aspectos filosóficos y comportamentales que consisten en tres componentes: orientación al cliente, orientación a la competencia y coordinación interfuncional. A su vez, Kohli y Jaworski (1990) 
defienden la orientación al mercado desde una perspectiva conductual de procesamiento de información; de generación de información procedente del mercado (para comprender sus necesidades), diseminación de dicha información (para compartir esa compresión), y de respuesta a la misma (para la satisfacción de las necesidades del mercado). Constituyéndose dicho procesamiento de información como el núcleo central de la orientación al mercado, tanto en el ámbito lucrativo como en el ámbito sin ánimo de lucro, en el sector privado y en el sector público (Cervera, 2004). De hecho, durante los últimos años la adopción de los planteamientos teóricos de la orientación al mercado han mostrado una gran eficacia al aplicarlos a instituciones públicas (Flavián y Lozano, 2004).

Además de que las características propias de las instituciones/organizaciones públicas son o deben ser argumentos suficientes para justificar la necesidad de la aplicación de la orientación al mercado para la mejora de su objeto de servicio al público, puesto que, ésta es el que legitima su existencia (Hayden, 1993), diversas experiencias en el ámbito del sector público apoyan la aplicación de determinadas herramientas de marketing como consecuencia de la adopción de una filosofía de acercamiento al público y, así, satisfacer las necesidades del mismo de manera más eficiente en lo que se ha denominado Orientación al Servicio del Público (Cervera, 1999).

Al considerarse que es apropiado desarrollar medidas del concepto adaptado a cada contexto específico, y por tanto, también, en el ámbito de la Administración Pública como organización no lucrativa proveedora directa o indirecta de servicios, considerando las peculiaridades del modelo para ella (Diamantopoulos y Hart, 1993 y Cadogan y Diamantopoulos, 1995). Se ha convertido el modelo de Kohli y Jaworski adaptado por otros autores en el ámbito no lucrativo (Bhuian, 1992; Wood y Bhuian, 1993; Bennett, 1998; Caruana et a., 1998; Wood et al, 2000; Váquez et al, 2002; Flavián y Lozano, 2007; y Agirre et al, 2014) como el modelo más aplicado en dicho ámbito y por tanto, idóneo para el análisis de la orientación al mercado en la Administración Pública: Orientación al Servicio del Público (Cervera, 1999 y 2004).

Así, en base a Cervera (1999 y 2004) se identifica los ítems representando las dimensiones del concepto de orientación al servicio del público (véase Tabla 1.1) creando así la escala de orientación al servicio del público y se establece una propuesta de modelo de Orientación al Servicio del Público (véase Figura 1.3). 


\section{Tabla 1.1}

Escala de Orientación al Servicio del Público

\section{Generación de información}

G1* Esta dirección no conoce la opinión que tiene su público objetivo de los servicios públicos que presta

G2 En esta dirección se recoge y analiza información sobre las necesidades aún no cubiertas del público objetivo

G3 Esta dirección recoge y analiza información sobre el impacto que pueden tener determinadas decisiones sobre el público objetivo

G4 Esta dirección recoge y analiza información sobre los servicios prestados y sus beneficiarios.

G5 Esta dirección consulta públicamente a través de reuniones abiertas o con los grupos que pueden verse afectados

G6* En esta dirección no se acude a actos (jornadas, congresos, seminarios, etc.) periódicamente para acercarse al público y conocer sus necesidades

G7* Esta dirección no conoce las necesidades de los grupos cuya voz se escucha con menor frecuencia (pequeñas entidades no asociadas a organizaciones representativas)

G8* Esta dirección no dedica tiempo y esfuerzo a comprender las necesidades del público objetivo

G9* Esta dirección no dedica tiempo y esfuerzo a comprender cómo los factores ambientales influyen en las necesidades del público objetivo

G10 En esta dirección se organizan discusiones con grupos de usuarios y no usuarios de los servicios para conocer actitudes y comportamientos difícilmente captados por las encuestas

G11* En esta dirección no se conoce la satisfacción de los beneficiarios de los servicios que presta

G12* Esta dirección es lenta en detectar los cambios de preferencias del público objetivo

G13* Esta dirección es lenta en detectar los cambios del entorno que le rodea

G14 En esta dirección se realizan periódicamente encuestas para conocer aspectos del sector y sus miembros

G15 Esta dirección conoce lo que otras unidades administrativas análogas realizan para satisfacer al público

\section{Diseminación de la información}

D1 Esta dirección se asegura de que la información sobre el público sea comunicada a toda la organización

D2 Esta dirección se coordina con las demás unidades administrativas inmersas en la prestación de un mismo servicio al público objetivo

D3 El personal de contacto con el público tiene los cauces formales adecuados para comunicar los problemas que le surgen en su labor cotidiana

D4 Cuando ocurre algo que afecta al público objetivo todo el personal de la dirección se entera rápidamente

D5 Muchas conversaciones informales entre el personal de esta dirección se refieren a las necesidades del público objetivo 


\section{Respuesta a la información}

R1* En esta dirección, en el diseño del servicio público no se consideran las demandas y necesidades del público objetivo

R2* En esta dirección, en el diseño del servicio público no se considera la información sobre los servicios prestados y sus beneficiarios

R3* En esta dirección no se provee información al público objetivo sobre su derecho a recibir servicios

R4* En esta dirección no se provee información al público objetivo sobre cómo acceder a un servicio y utilizarlo

R5* Esta dirección no utiliza un lenguaje y unos medios sencillos y claros para comunicarse con el público objetivo

R6* En esta dirección no se facilita el contacto al público con la persona que necesita en el instante que la necesita

R7* Esta dirección no posee unos buenos canales de comunicación con el público

R8* Esta dirección no fomenta la participación del público objetivo en la vida política

R9* En esta dirección las quejas del público caen en saco roto

R10 En esta dirección la información sobre el público objetivo se utiliza en el proceso de elaboración de presupuestos

R11 Esta dirección es transparente en la toma de decisiones y en la gestión

R12 En esta dirección se flexibilizan los horarios para adaptarse a las necesidades del público

R13 En esta dirección se da la información al público que realmente le interesa, en el momento y en el lugar adecuados

R14 En esta dirección se provee de información al público objetivo sobre los servicios públicos disponibles y no disponibles y el por qué lo están o no

R15 Esta dirección informa al público objetivo sobre los niveles de prestación de servicios que se pretende alcanzar ( $\mathrm{n} .^{\circ}$ de beneficiarios a atender, etc.)

R16 En esta dirección se informa al ciudadano de cómo hacer quejas, qué ocurre tras su realización, y el tiempo de espera estimado hasta obtener respuesta

$(*)$ : Indica ítem en sentido inverso.

Fuente: elaboración propia.

Respecto a las consecuencias (véase Tabla 1.2), se ha llevado a cabo el mismo proceso de creación y adaptación de la escala de las consecuencias de la orientación al servicio del público. Cabe apuntar que respecto a la participación se ha dividido cada ítem relacionado con la participación en dos en base a Young (1991), que toma en consideración la participación tanto de los ciudadanos como los representantes de éstos 
en los procesos de planificación y prestación de los servicios para que una Administración Pública esté orientada. Pero también teniendo en cuenta diversos trabajos sobre la participación ciudadana o participación pública (Thomas, 1995 y 2012; Irvin y Stansbury, 2004 y Bryson et al, 2013) que hacen referencia no solo a los ciudadanos sino también a los stakeholders. En consecuencia, se han incorpora los ítmes C8, C10 y C12.

\section{Tabla 1.2}

Consecuencias de la Orientación al Servicio del Público

C1 El tiempo de prestación del servicio es

C2 Las relaciones entre funcionarios y los políticos es

C3 El personal de la dirección tiene un sentimiento de pertenencia

C4 La satisfacción del público es

C5 La calidad de los servidos prestados por la dirección es

C6 La atención al público de la dirección es

C7 La participación del público en la planificación de nuevos servicios es

C8 La participación de las organizaciones representativas del público en la planificación de nuevos servicios es

C9 La participación del público en los procesos de modificación o eliminación de servicios es

C10 La participación de las organizaciones representativas del público en los procesos de modificación o eliminación de servicios es

C11 La participación del público en la prestación del servicio es

C12 La participación de las organizaciones representativas del público en la prestación del servicio es

Fuente: elaboración propia.

De esta manera, se propone como modelo que mejor podría explica la orientación al servicio del público aquel que está constituido por tres factores correlacionados: los tradicionales de generación, diseminación y respuesta a la información (Figura 1.3) 


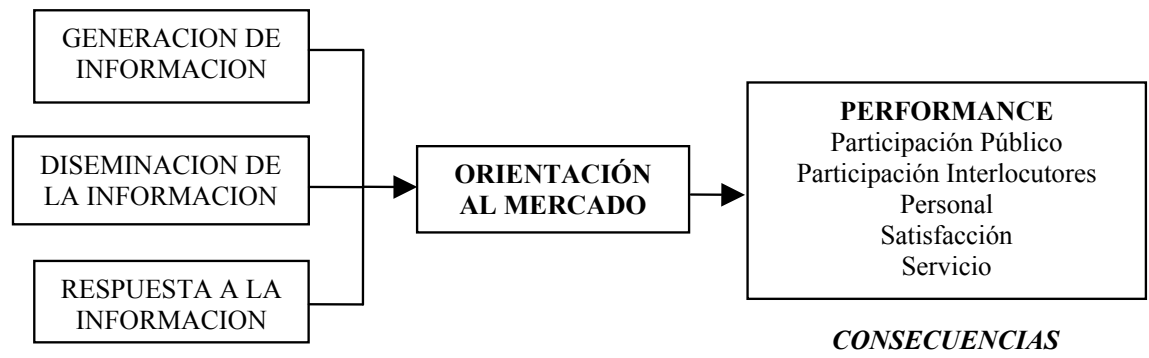

Fuente: elaboración propia.

\section{Figura 1.3}

Propuesta de Modelo de Orientación al Servicio del Público

\section{Estudio de caso}

\section{Objeto de estudio}

Atendiendo a la distinción de niveles desde la perspectiva del Marketing Público, entre Marketing Público Institucional y Marketing de Servicios Públicos, y a que el nivel de orientación al mercado varía entre los diferentes niveles de una misma organización empresarial (Ruekuert, 1992), resulta de interés el análisis de la cuestión en la unidad básica de actuación pública de los gobiernos.

En este caso, se ha optado por la unidad administrativa básica de actuación pública para el fomento de la Economía Social en el País Vasco: la Dirección de Economía Social del Gobierno Vasco. Es éste el órgano institucional dedicado al fomento y promoción de la Economía Social vasca, es decir, es el agente por excelencia de promoción de la Economía Social vasca a la que le presta servicios públicos de fomento. Por tanto, el objetivo es el análisis de la Orientación al Servicio del Público y sus consecuencias en la Dirección de Economía Social del Gobierno Vasco, ya que es ésta la unidad básica de actuación en el ámbito de fomento (Servicio de Fomento) de la Economía Social.

\section{Metodología}

Se han utilizado las escalas citadas anteriormente aplicando escalas Likert de 5 en las que se incluyen ítems en sentido inverso para evitar erro- 
res de tendencia en las respuestas o vicios de mero consentimiento, o sesgo de aquiescencia/asentimiento ${ }^{3}$.

Con todo ello, se han realizado entrevistas en profundidad semiestructuradas, se ha utilizado un guión con los temas a tratar en la entrevista y controlando directamente el proceso de obtención de información, lo que ha permitido obtener información a través de conversaciones personales e individualizadas (Fernández, 1999: 207 y 210). Es decir, se han realizado entrevistas personales teniendo como guión de la entrevista la propia encuesta, dado que además de responder una encuesta se pretendía obtener informaciones cualitativas difíciles de captar mediante las encuestas, y así complementarlas. Siguiendo una de las tendencias en técnicas cualitativas de combinación de metodologías denominada por Fernández (1999: 207) Técnica cualitativalcuantitativa, se ha desarrollado la entrevista aplicando las escalas.

Para la recogida de datos se ha utilizado el método del «informanteclave» (Campbell, 1955) que se basa en la existencia de individuos en una organización con determinadas aptitudes, status y amplio conocimiento que proveen información de la colectividad.

El universo de referencia para la encuesta y entrevista a efectos de medición del nivel de orientación al servicio del público de la Dirección de Economía Social del Gobierno Vasco ha sido la propia Dirección, sirviéndonos como informante-claves de los técnicos del área de fomento de la misma $a^{4}$. Se han seleccionado estos informantes dado que todos ellos se encuentran en una buena posición para poder comprender y describir las características y conceptos organizativos que se tratan, y por su independencia (laboral) con respecto a los partidos políticos.

Para la determinación del grado o nivel de orientación al servicio del público, se suele plantear la utilización de las puntuaciones de los factores o de las variables, teniendo ambas ventajas y desventajas, sin existir una única respuesta para todas las situaciones (Cervera, 1999 y 2004). Las puntuaciones de los factores tienen la ventaja de representar una composición de todas las variables que cargan en el factor mientras que las variables representan una sola variable. Pero dado que las puntuaciones de los factores están basadas en correlaciones con todas las variables en el factor y estas co-

3 Este sesgo designa la tendencia de los sujetos a expresar acuerdo con el ítem, independientemente del contenido del mismo (Ray, 1990), o tendencia de responder siempre afirmativamente. En base a Cervera et al (1999) siguiendo a Gil (1995) y las matizaciones de Carman (1990) se incluyen ítems en sentido negativo.

4 Técnicos responsables de la competencia atribuida a la Dirección de Economía Social para la gestión de los programas de fomento de la Economía Social, es decir, de las subvenciones dirigidas Economía Social (Bakaikoa y Morandeira, 2012). 
rrelaciones serán probablemente menores que la unidad, las puntuaciones son solo aproximaciones de los factores y como tales indicadores de los factores subyacentes expuestos a error, pero sigue siendo la mejor de las alternativas en el caso de escalas bien construidas, fiables y válidas (Hair, et al, 2007).

En este caso, para el grado o nivel de orientación al servicio del público y sus consecuencias en la Dirección de la Economía Social del Gobierno Vasco, al no estar la escala testada y con poca evidencia de fiabilidad y validez se utilizaran las variables (Cervera, 1999).

Por tanto, en primer lugar, se calculan los niveles medios de orientación al servicio del público por medio de las medias y teniendo en cuenta las desviaciones típicas en cada uno de los ítems incluidos en los factores/dimensiones.

En segundo lugar, pudiendo ser las técnicas cualitativas y las cuantitativas procedimientos complementarios, se desarrolla un análisis cualitativo de los aspectos de la orientación al servicio del público 5 . El estudio cualitativo proporciona información de gran utilidad para la interpretación de los resultados de las técnicas cuantitativas y, a su vez, proveyendo a los datos cuantitativos mayor valor cuando están arropadas con una información cualitativa que aporta una justificación de dichos datos (Fernández, 1999). Es más, «la combinación de enfoques cuantitativos y cualitativos permite conseguir un conocimiento más completo del tema estudiado.» (Ortega, 2010: 143).

Y por último, se creará un indice de medida del nivel de orientación al servicio del público de la Dirección de Economía Social a partir de las variables/ítems (Gaski y Etzel, 1986).Con el objeto de hacer más comprensible dicha puntuación y dado que cada uno de los ítems puede llegar a tener una puntuación entre 1 y 5 puntos, es decir, son variables medidas en una escala Likert de 5, se han generado cuatro intervalos (Nada orientado, poco orientado, bastante orientado y muy orientado) que determinan los diferentes posibles niveles de orientación al servicio del público y sus consecuencias.

\section{Análisis de resultados}

En relación, al grado o nivel de orientación al servicio del público, se calculan los niveles medios de orientación al servicio del público por medio

5 No se ha incluido análisis cualitativo en relación a las consecuencias por lo que los entrevistados no han estimado oportuna realizar matizaciones o aportaciones más allá de la puntuación que se ha señalado. 
de las medias y teniendo en cuenta las desviaciones típicas en cada uno de los ítems incluidos en los factores/dimensiones.

\section{Tabla 1.3}

Medias y desviaciones de los ítems de las dimensiones de la Orientación al Servicio del Público

\begin{tabular}{|c|c|c|c|}
\hline Ítems & Media & Desviación & Dimensión \\
\hline G1* & 4 & 0 & \multirow{15}{*}{$\underset{3,63}{\text { GI }}$} \\
\hline G2 & 3,5 & 0,5 & \\
\hline G3 & 3,5 & 0,5 & \\
\hline G4 & 4 & 0 & \\
\hline G5 & 3,5 & 0,5 & \\
\hline G6* & 4,5 & 0,5 & \\
\hline $\mathrm{G} 7^{*}$ & 3 & 1 & \\
\hline G8* & 4,5 & 0,5 & \\
\hline G9* & 4 & 1 & \\
\hline G10 & 3 & 1 & \\
\hline G11* & 4 & 0 & \\
\hline G12* & 3 & 0 & \\
\hline G13* & 3,5 & 0,5 & \\
\hline G14 & 2,5 & 0,5 & \\
\hline G15 & 4 & 0 & \\
\hline D1 & 4 & 1 & \multirow{5}{*}{$\begin{array}{l}\text { DI } \\
3,7\end{array}$} \\
\hline D2 & 3,5 & 0,5 & \\
\hline D3 & 4,5 & 0,5 & \\
\hline $\mathrm{D} 4$ & 3,5 & 0,5 & \\
\hline D5 & 3 & 0 & \\
\hline
\end{tabular}

\begin{tabular}{|c|c|c|c|}
\hline Ítems & Media & Desviación & Dimensión \\
\hline $\mathrm{R} 1^{*}$ & 5 & 0 & \multirow{16}{*}{$\underset{4,25}{\mathrm{RI}}$} \\
\hline R2* & 5 & 0 & \\
\hline R3* & 5 & 0 & \\
\hline $\mathrm{R} 4^{*}$ & 4,5 & 0,5 & \\
\hline $\mathrm{R} 5^{*}$ & 4 & 0 & \\
\hline R6* & 4 & 0 & \\
\hline $\mathrm{R}^{*}$ & 4 & 0 & \\
\hline $\mathrm{R} 8 *$ & 3 & 2 & \\
\hline R9* & 5 & 0 & \\
\hline $\mathrm{R} 10$ & 4,5 & 0,5 & \\
\hline R11 & 4 & 1 & \\
\hline $\mathrm{R} 12$ & 3,5 & 0,5 & \\
\hline R13 & 4 & 0 & \\
\hline $\mathrm{R} 14$ & 4 & 0 & \\
\hline R15 & 4 & 0 & \\
\hline R16 & 4,5 & 0,5 & \\
\hline
\end{tabular}

GI: Generación de información.

DI: Diseminación de la información.

RI: Respuesta a la información.

Fuente: Elaboración propia. Tratamiento de datos Dyane versión 4. 
Se observa (véase Tabla 1.3) que el factor o dimensión que mayor puntuación obtiene es el de respuesta la información con una puntuación media de un 4,25; seguido por diseminación de la información con un 3,7; y por generación de información con un 3,63.

A continuación, se realiza un análisis más exhaustivo para cada escala, es decir, para cada dimensión del constructo orientación al servicio del público, valiéndonos de la información obtenida por medio de las encuestas, es decir, un análisis cualitativo:

- En cuanto a la generación de información, la Dirección de Economía Social muestra una tendencia a trabajar con los interlocutores. Es decir, son los interlocutores/intermediarios (organizaciones representativas y promotoras) los canalizadores de todo, o casi todo el flujo de información que recibe la Dirección respecto al público objetivo.

- Respecto a la diseminación de la información se destaca la influencia del tamaño de la unidad en este ámbito, ya que es muy citado en las entrevistas. De hecho, el tamaño reducido de la Dirección es un factor facilitador de la comunicación dentro de la misma.

-Y en último lugar, en cuanto a la respuesta a la información se puede destacar que está condicionada por el tamańo de la unidad, recursos económicos.

Por otro lado, se ha creado un indice de medida del nivel de orientación al servicio del público de la Dirección de Economía Social (Gaski y Etzel, 1986), se han obtenido las siguientes puntuaciones (véase Tabla 1.4), clasificadas respecto a cuatro intervalos, para cada uno de los constructos definidos aunque en este trabajo se ha tenido en consideración el último de ellos.

\section{Tabla 1.4}

Nivel de Orientación al Servicio del Público de la Dirección de Economía Social del Gobierno Vasco

\begin{tabular}{|c|c|c|c|c|c|}
\hline \multirow[b]{2}{*}{ Constructos } & \multirow[b]{2}{*}{ Puntuación } & \multicolumn{4}{|c|}{ Categoría } \\
\hline & & $\begin{array}{c}\text { Nada } \\
\text { Orientada }\end{array}$ & $\begin{array}{c}\text { Poco } \\
\text { Orientada }\end{array}$ & $\begin{array}{c}\text { Bastante } \\
\text { Orientada }\end{array}$ & $\begin{array}{c}\text { Muy } \\
\text { Orientada }\end{array}$ \\
\hline OSP & $36-180$ & $X$ & $X$ & 141 & X \\
\hline
\end{tabular}

Fuente: Elaboración propia.

Se observa que la Dirección de Economía Social del Gobierno Vasco está bastante orientada al servicio del público. Aunque es de señalar que la 
generación de información es la dimensión que más dudas surge por el valor medio alcanzado, que se corresponde con el análisis cualitativo de las encuestas en el que se subraya a los interlocutores como canalizadores y emisores de la información que recibe la Dirección.

Atendiendo al estudio de las consecuencias de la orientación al servicio del público, se han calculado, también, los niveles medios de las mismas por medio de las medias y teniendo en cuenta las desviaciones típicas en cada uno de los ítems incluidos.

Si bien se ha determinado que no existen diferencias significativas entre los valores medios de las dos muestras, trabajadores/técnicos de la Dirección de Economía Socia y representantes de las organizaciones representativas de las entidades de Economía Social, se muestran los datos de manera conjunta y separada ya que en el caso de los informantes de la Dirección de Economía Social incluían dos ítems que no procedía incluir para los representantes de la organizaciones representativas y que son de interés.

Así, en primer lugar, respecto a las consecuencias valoradas por los informante-claves de la Dirección se han obtenido los valores que se muestran en la Tabla 1.5.

\section{Tabla 1.5}

Medias y desviaciones de los ítems de las dimensiones de las consecuencias I

\begin{tabular}{|c|c|c|c|}
\hline Ítems & Media & Desviación & Consecuencias \\
\hline $\mathrm{C} 4$ & 3,5 & 0,5 & $\begin{array}{c}\text { Satisfacción } \\
3,5\end{array}$ \\
\hline $\mathrm{C} 2$ & 4 & 1 & Personal \\
\hline $\mathrm{C} 3$ & 3,5 & 0,5 & 3,75 \\
\hline $\mathrm{C} 1$ & 2 & 0 & \\
\hline $\mathrm{C} 5$ & 4 & 0 & $\begin{array}{l}\text { Servicio } \\
35\end{array}$ \\
\hline C6 & 4,5 & 0,5 & \\
\hline C7 & 1,5 & 0,5 & Participación \\
\hline C9 & 1,5 & 0,5 & Público \\
\hline $\mathrm{C} 11$ & 1,5 & 0,5 & 1,5 \\
\hline $\mathrm{C} 8$ & 4 & 1 & Participación \\
\hline $\mathrm{C} 10$ & 4 & 1 & Interlocutores \\
\hline $\mathrm{C} 12$ & 4,5 & 0,5 & 4,16 \\
\hline
\end{tabular}

Fuente: Elaboración propia. Tratamiento de datos Dyane versión 4. 
Se observa que el factor o dimensión que mayor obtiene es la que hace referencia a la participación de los interlocutores u organizaciones representativas (con una puntuación media de 4,16), seguida por las consecuencias sobre el personal $(3,75)$, con un 3,5 para la satisfacción y características del servicio y, por último, con una puntuación de 1,5 para la participación del público.

Por otro lado, siguiendo lo expuesto en el apartado de metodología sobre la creación de un indice de las consecuencias y teniendo en cuenta la necesidad de considerar de forma separada las dimensiones del performancel desempeño/consecuencias (Jaworski y Kohli, 1996), se han obtenido las siguientes puntuaciones (Tabla 1.6) clasificadas respecto a cuatro intervalos.

Tabla 1.6

Nivel de las consecuencias de la Orientación al Servicio del Público de la Dirección de Economía Social del Gobierno Vasco

\begin{tabular}{lccccc}
\hline & & \multicolumn{4}{c}{ Categoría } \\
\cline { 3 - 6 } \multicolumn{1}{c}{ Dimensiones } & Puntuación & $\begin{array}{c}\text { Nada } \\
\text { Bueno/a }\end{array}$ & $\begin{array}{c}\text { Poco } \\
\text { Bueno/a }\end{array}$ & $\begin{array}{c}\text { Bastante } \\
\text { Bueno/a }\end{array}$ & $\begin{array}{c}\text { Muy } \\
\text { Bueno/a }\end{array}$ \\
\hline Satisfacción & $1-5$ & $\mathrm{X}$ & $\mathrm{X}$ & $\mathbf{3 , 5}$ & $\mathrm{X}$ \\
Servicio & $3-15$ & $\mathrm{X}$ & $\mathrm{X}$ & $\mathbf{1 0 , 5}$ & $\mathrm{X}$ \\
Participación Público & $3-15$ & $\mathbf{4 , 5}$ & $\mathrm{X}$ & $\mathrm{X}$ & $\mathrm{X}$ \\
Participación Interlocutores & $3-15$ & $\mathrm{X}$ & $\mathrm{X}$ & $\mathrm{X}$ & $\mathbf{1 2 , 5}$ \\
Personal & $2-10$ & $\mathrm{X}$ & $\mathrm{X}$ & $\mathbf{7 , 5}$ & $\mathrm{X}$ \\
\hline
\end{tabular}

Fuente: Elaboración propia.

Tal y como se puede observar, la Dirección de Economía Social del Gobierno Vasco, con un nivel de orientación al mercado bastante alto, logra un performance bastante bueno en términos generales. Pero atendiendo a que es necesario separar las dimensiones del performance/desempeño/consecuencias (Jaworski y Kohli, 1996), hay que señalar que la participación del público no llega a ser aceptable.

\section{Discusión e implicación}

Teniendo en cuenta que la orientación al mercado puede variar entre distintos niveles de una misma organización y que la mayor parte de los estudios realizados, sino todos, en materia de orientación al servicio 
del público se han centrado en el ámbito institucional o nivel jerárquico de decisión estratégica, este trabajo se ha concentrado en el análisis de la orientación al servicio del público en una unidad básica de actuación pública dentro de un Gobierno, es decir, en un nivel jerárquico de la esfera táctica y operativa de la actuación pública. Así, el objetivo principal del trabajo ha sido analizar la orientación al servicio público en el ámbito del sector público de la unidad básica de actuación pública de fomento de la Economía Social vasca.

Se observa que la Dirección de Economía Social está bastante orientada al servicio del público. Es decir, la Dirección de Economía Social del Gobierno Vasco tiene un nivel medio-alto de orientación al servicio del público, aunque, si se observa que la generación de información es la dimensión en la que más dudas surgen, que se corresponde con el análisis cualitativo en el que se subraya a los interlocutores como canalizadores y emisores de la información que recibe la Dirección y, por tanto, no genera excesiva información de manera directa respecto al público. En la diseminación de la información, se destaca la influencia del tamaño reducido de la unidad como un factor facilitador de la comunicación dentro de la misma, y en la respuesta a la información las dificultades por la escasez de recursos y los procedimientos a seguir en el ámbito público aunque con una apuesta inequívoca a la provisión de información.

Por último, del análisis de las consecuencias de la orientación al servicio del público de la Dirección de Economía Social, se observa que todas las dimensiones consideradas como consecuencia han sido valoradas muy positivamente excepto la participación del público con una valoración escasa, ya que aunque el público participa, lo hace por medio de los interlocutores. Resultado este que muestra la idoneidad del planteamiento teórico propuesta a la hora de diferenciar los dos niveles de participación, ya que unidas en una misma variable no se hubiera podido advertir dicha diferencia a no ser que los entrevistados la plantearan en la entrevista.

Por tanto, aunque sin poder establecer una relación de causalidad, podemos afirmar que la unidad básica de actuación básica de fomento de la Economía Social que tiene un alto nivel de orientación al servició del público, logra un buen rendimiento en cuanto satisfacción, personal, servicio y participación.

\section{Bibliografía}

Aguirre, I.; Reinares, P. and Agirre, A. (2014): «Antecedents to market orientation in the worker cooperative organization: The Mondragon Group", Annals of Public and Cooperative Economics, Vol.: 85:3, pp. 387-408. 
Bakaikoa, B. y Morandeira, J. (2012): «El cooperativismo y las políticas públicas», Revista Vasca de Economía-Ekonomiaz, Vol. 79: 1, pp. 233-261.

Bennett, R. (1998): "Charities, organizational learning and market orientation», Journal of Marketing Practice, Vol. 4: 1, pp. 5-16.

Bhuian, S. N. (1992): Market orientation and Successful Not-For-Profit Marketing: Direct Relationship and the Influence of Professionalism, Entrepreneurship, Competition and Demand. Tesis Doctoral, Texas Tech University.

Bouinot, J. y Bermils, B. (1995): La gestión stratégique des villes: entre compétition et coopération. Editions Armand Colin, Paris.

Boulding, K.E. (1956): "General Systems Theory-The Skeleton of Science», Management Scince, Abril.

Bryson, J.M.; Quick, K.S.; Slotterback C.S. y Crosby, B.C. (2013): «Designing Public Participation Process: Theory to Practice», Public Administration Review, Vol. 73: 1, pp. 23-34.

Cadogan, J.W. y Diamantopoulos, A. (1995): «Narver and Salter, Kohli and Jaworski and the Market Orientation Construct: Integration and Internationalization. Journal of Strategic Management, Vol. 3: 1, pp. 41-60.

Campbell, D.T. (1955): "The informant in quantitative research», American Journal of Sociology, n. ${ }^{\circ} 60$, pp. 339-342.

Carman, J.M. (1990): "Consumer Perceptions of Service Quality: An Assement of the Servqual Dimensions», Journal of Retailing, Vol. 66: 1, pp. 33-55.

Caruana, A.; Ramaseshan, B. y Ewing, M.T. (1998): "The Marketing Orientation-Performance Link: Some evidence from the Public Sector and Universities", Journal of Nonprofit and Public Sector Marketing, Vol. 6: 1, pp. 63-82.

Cervera, A. (1999): "Construcción de una modelo de orientación al mercado en las Administraciones Públicas», Investigaciones Europeas de Dirección y Economía de la Empresa, Vol. 5: 3, pp. 41-62.

Cervera, A. (2001): «La gestión del marketing en la administración pública local». Dirección y organización: Revista de Dirección, Organización y Administración de empresas, n. ${ }^{\circ}$ 26, pp. 113-124.

Cervera, A. (2004): «Orientación al mercado y Administración Pública Local: Determinación de una modelo teórico y de medición», Revista Internacional de Marketing Público y No Lucrativo, Vol. 1: 1, junio, pp. 53-72.

Cervera, A.; Mollá, A. y Calderón H. (1999): «Market Orientation: A Framework for Public Institutions», Journal of Nonprofit \& Public Sector Marketing, Vol. 7: 1, pp. 3-23.

Cervera, A.; Mollá, A. y Sánchez, M. (2001): «Antecedents and consequences of market orientation in public organisations», European Journal of Marketing, Vol. 35: 11/12, pp. 3-23.

Chias, J. (1995): Marketing Público. Por un Gobierno y una Administración al servicio del público, McGraw-Hill Interamericana de España, Madrid.

Chias, J. (2007): «Marketing Público en España y Latinoamérica: algunas consideraciones» en Kotler, P. y Lee, N.: Marketing en el Sector Público. Todas las claves para su mejora, Pearson Educación, Madrid, pp. 489-499. 
Cowell, D. (1991): Mercadeo de Servicios. Legis, Bogotá.

Diamantopolous, A. y Hart, S. (1993): «Linking Market Orientation and Company Performance: Preliminary Evidence on Kohli and Jaworski's Framework», Journal of Strategic Management, Vol. 1: 2, pp. 93-121.

Fernádez, A. (1999): "La investigación cualitativa», en Sarabia, F.J.: Metodología para la investigación en marketing y dirección de empresas, Pirámide, Madrid.

Flavián, C. y Lozano, F.J. (2004): «La orientación al mercado de la universidad pública: Un reto para el sistema universitario español», Revista Internacional de Marketing Público y No Lucrativo, Vol. 1: 2, pp. 9-28.

Flavián, C. y Lozano, F.J. (2007): «Market Orientation of Spanish Public Universities: A suitable Response to Growing Competition», Journal of Marketing of Higher Education, Vol. 17: 1, pp. 91-116.

Gaski, J.F. y Etzel, M.J. (1986), «The index of consumer sentiment toward marketing», Journal of Marketing, Vol. 50: 3, pp. 71-82.

Gil, I. (1995): La calidad de servicio percibida y la selección del punto de venta, Club Gestión de Calidad, Madrid.

Grönroos, C. (1989): «Defining Marketing: A Market-Oriented Approach». European Journal of Marketing, Vol. 23: 1, pp. 52-60.

Hair, J.F.; Black W.C.; Babin B.J. y Anderson R.E. (2009): Multivariate Data Analysis (7th Edition), Prentice Hall, New Jersey.

Hayden, V. (1993): «How to increase market orientation», Journal of Management in Medicine, Vol. 7: 1, pp. 29-46.

Irvin, R.A. y Stansbury, J. (2004): "Citizen Participation in Decision Making: Is It Worth the Effort?», Public Administration Review, Vol. 64: 1, pp. 55-65.

Jaworski, B. y Kohli, A. K. (1996): "Market Orientation: Review, Refinement, and Roadmap,» Journal of Market-Focused Management, Vol.: 1: 2, 119-35.

Kohli, A. K. y Jaworski, B.J. (1990): "Market Orientation: The Construct, Research Propositions and Managerial Implications». Journal of Marketing, Vol. 54: 2, pp. 1-18.

Kohli, A.K.; Jaworski, B.J. y Kumar, A. (1993): «MARKOR: A Measure of Market Orientation». Journal of Marketing Research, Vol. 30: 4, Noviembre, pp.467-477.

Kotler, P. y Lee, N. (2007): Marketing en el Sector Público. Todas las claves para su mejora, Pearson Educación, Madrid.

Lamb, C. (1987): «Public Sector Marketing is Different», Business Horizons, Vol.30: 4, pp. 46-54.

Lambin, J.J. (2003): Marketing estratégico, ESIC, Madrid.

Laufer, R. (1976): «Le marketing du service public», Revue Française de Gestion, Noviembre-Diciembre, pp. 72-87.

Laufer, R. y Burlaud, A. (1989): Dirección Pública: gestión y legitimidad. INAP, Madrid.

Martín, E. (1993): Marketing, Ariel, Barcelona. 
McCarthy, E.J. y Perreault, W.D. (1993): Basic Marketing: A Global-Managerial Approach, 11 ed., Irwin. Homewood, Illinois.

Miquel, S.; Bigné, E.; Sánchez, J.; Moliner, M.A. y Vallet, T. (1995): «El marketing de los servicios públicos administrativos», Congreso de AEDEM, pp. 22872299.

Narver, J.C. y Slater, S.F. (1990): «The Effects of a Market Orientation on Business Profitability». Journal of Marketing, Vol. 54: 4, pp.20-35.

Ortega, E. (2010): «La investigación en marketing a través de las tesis doctorales españolas", Investigaciones europeas de dirección y economia de la empresa, Vol.: 16:1, pp. 127-147.

Ramió, C. (1999): Teoría de la Organización y Administración Pública, Tecnos, Madrid.

Ray, J.J. (1990): "Acquiescence and problems with forced-choice scales», The Journal of Social Psychology, Vol. 130, pp. 397-399.

Ritchie, J.R.B. y La Brèque, R.J. (1975): "Marketing Research and Public Policy: A Functional Perspective», Journal of Marketing, Vol. 39: 3, pp. 12-19.

Röber, M. (1989): "Posibilidades y límites del marketing público. Notas desde una perspectiva de Ciencia Administrativa», Documentación Administrativa, Vol. 218-219, abril-septiembre, pp. 437-451

Rufín, R. y Medina, C. (2012): Marketing Público: Investigación, aplicaciones y estrategia, ESIC, Madrid.

Ruekert, R. W. (1992): «Developing a Market Orientation: An Organizational Strategy Perspective. International Journal of Research in Marketing, Vol. 9: 3, pp. 225-245.

Santesmases, M. (2009): Dyane Versión 4. Diseña y análisis de encuestas en investigación social y de mercados, Ediciones Pirámide, Madrid.

Santesmases, M. (2012): Marketing: Conceptos y estrategias. 6. ${ }^{a}$ edición, Ediciones Pirámide, Madrid.

Snavely, K. (1991): «Marketing in the Government Sector: A Public Policy Model», American Review of Public Administration, Vol. 21 (4), pp. 311-326.

Subirats, J. (1992): Un problema de estilo: la formación de politicas públicas en España., Centro de Estudios Políticos y Constitucionales, Madrid.

Thomas, J.C. (1995): Public Participation in Public Decision: New Skills and Strategies for Public Managers, Jossey-Bass, San Francisco.

Thomas, J.C. (2012): Citizen, customer, Partner: Engaging the Public in Public Management, M.E.Sharpe, Armonk (New York).

Vázquez, R.; Álvarez, L. I.; Santos, M. L. (2002): «Marketing orientation and social services in prívate non-profit organisations», European Journal of Marketing, Vol. 9/10, pp. 1022-1046.

Vázquez, J.L. y Placer, J.L. (2000): Cinco temas de introducción al marketing público, Imprenta Moderna, León.

Walsh, K. (1994): «Marketing and Public Sector Management», European Journal of Marketing, Vol. 28: 3, pp. 63-71. 
Wood, V.R.; Bhuian, S. (1993): «Market Orientation and Nonprofit Organizations: Performance Associations and Research Propositions». Journal of Nonprofit \& Public Sector Marketing, Vol. 1: 1, pp. 7-32.

Wood, V.R.; Bhuian, S. y Kieker, P. (2000): «Market orientation and organizational performance in Nom-for-Profit Hospitals», Journal of Business Research, Vol. 48, pp. 213-226.

Young, K. (1991): "Consumer-centred approaches in the public/voluntary/personal services», Public Money \& Management, Vol. 11: 2, pp. 33-39. 